\title{
Association of pregnancy-associated plasma protein A and vascular endothelial growth factor with pregnancy-induced hypertension
}

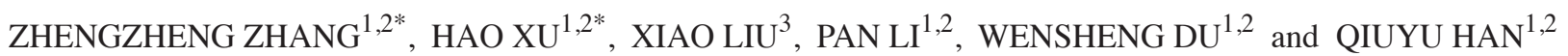 \\ ${ }^{1}$ Department of Obstetrics and Gynecology, The Affiliated Hospital of Xuzhou Medical University; \\ ${ }^{2}$ Xuzhou Medical University; ${ }^{3}$ Department of Emergency, The Affiliated Hospital \\ of Xuzhou Medical University, Xuzhou, Jiangsu 221002, P.R. China
}

Received January 29, 2019; Accepted May 10, 2019

DOI: $10.3892 /$ etm.2019.7724

\begin{abstract}
The present study aimed to evaluate changes of pregnancy-associated plasma protein A (PAPP-A) and vascular endothelial growth factor (VEGF) in pregnancy-induced hypertension (PIH). A total of 105 cases (observation group) with complete data that underwent delivery and suffered from PIH in The Affiliated Hospital of Xuzhou Medical University from February 2015 to February 2017 were retrospectively analyzed. The observation group was further divided into the mild observation and severe observation groups according to severity degree of the disease. Another 65 asymptomatic pregnant women were recruited as the healthy control group. Basic data, obstetric data, PAPP-A and VEGF and data of perinatal infants were compared and analyzed. The Logistic regression model was adopted to screen out risk factors for PIH. In the observation group, the rate of periodic antenatal care was lower, and there were more primigravidas and housewives, with lower education level and economic income $(\mathrm{P}<0.05)$. In the observation group, the occurrence rates of placental abruption as well as turbid and bloody amniotic fluid were higher than those in the healthy control group $(\mathrm{P}<0.05)$. The neonatal birth weight was lower in the observation group than that in the healthy control group, while the occurrence rates of neonatal department transfer, small for gestational age (SGA), neonatal asphyxia and
\end{abstract}

Correspondence to: Dr Wensheng Du or Dr Qiuyu Han, Department of Obstetrics and Gynecology, The Affiliated Hospital of Xuzhou Medical University, 99 Huaihai Xi Road, Xuzhou, Jiangsu 221002, P.R. China

E-mail: dws111@126.com

E-mail: mpia246@163.com

\section{${ }^{*}$ Contributed equally}

Key words: pregnancy-induced hypertension, pregnancy-associated plasma protein A, vascular endothelial growth factor, risk factor survival rates of perinatal infants were higher $(\mathrm{P}<0.05)$. PAPP-A levels at 34-40 gestational weeks in the observation group were significantly higher than those in the healthy control group $(\mathrm{P}<0.05)$. VEGF levels were lower than those in the healthy control group $(\mathrm{P}<0.05)$. Multivariate analysis revealed that high PAPP-A value [odds ratio $(\mathrm{OR})=3.736$ ] and identity of housewife $(\mathrm{OR}=2.514)$ were risk factors for $\mathrm{PIH}$, while high VEGF value $(\mathrm{OR}=5.258)$, non-primigravid $(\mathrm{OR}=2.173)$, higher economic income $(\mathrm{OR}=4.162)$ and periodic antenatal care $(\mathrm{OR}=1.201)$ were regarded as protective factors. Therefore, enhancement of gestational management, early discovery and early treatment are critical for improving the prognosis of pregnant women and infants.

\section{Introduction}

Pregnancy-induced hypertension (PIH) syndrome, is a disease that only occurs during pregnancy, and the basic pathophysiological changes include systemic small-vessel spasm and reduced perfusion of organs in each system (1). Besides, the disease refers to a symptom complex mainly manifested as hypertension, proteinuria, edema and multiple organ dysfunctions in clinical practice, which tends to result in poor prognoses such as fetal distress, fetal growth restriction (FGR) and premature birth, thus severely jeopardizing the maternal and neonatal health, so it is still one of the main causes of their death (2). Prior to the appearance of clinical symptoms in patients with $\mathrm{PIH}$, some biochemical changes tend to occur, so the incidence and mortality rates of PIH are expected to become lower by effective clinical predictors. Studies have demonstrated that pregnancy-associated plasma protein A (PAPP-A) is closely associated with the prediction, judgment of disease and prognosis of PIH $(3,4)$. According to other studies, changes in the expression of vascular endothelial growth factor (VEGF) may exert crucial effects in the pathogenesis of PIH $(5,6)$. Thus, this study investigated the association of PAPP-A and VEGF with the incidence of PIH by measuring their changes in maternal serum, so as to explore the main risk factors for $\mathrm{PIH}$, thus providing a basis for predicting $\mathrm{PIH}$ and improving maternal and neonatal outcomes. 
Table I. Comparison of basic data of pregnant women between the observation and healthy control groups.

\begin{tabular}{|c|c|c|c|c|}
\hline Observational indexes & Observation group $(\mathrm{n}=105)$ & Control group $(n=65)$ & $t / \chi^{2}$ & $\mathrm{P}$-value \\
\hline Regular antenatal examination & & & 11.052 & $<0.001$ \\
\hline Yes & 23 & 35 & & \\
\hline No & 82 & 30 & & \\
\hline Age (years) & $28.16 \pm 4.85$ & $27.84 \pm 5.02$ & 1.165 & 0.213 \\
\hline Gravidity (times) & $1.16 \pm 0.85$ & $2.19 \pm 1.21$ & 6.192 & 0.005 \\
\hline Parity (times) & $1.08 \pm 0.34$ & $1.56 \pm 1.03$ & 3.873 & 0.012 \\
\hline Gestational week (week) & $37.12 \pm 0.48$ & $37.09 \pm 0.53$ & 1.135 & 0.238 \\
\hline Conception method & & & 0.594 & 1.023 \\
\hline Natural & 95 & 59 & & \\
\hline ART & 10 & 6 & & \\
\hline Occupation & & & 7.948 & 0.003 \\
\hline Housewife & 66 & 20 & & \\
\hline Individual & 14 & 5 & & \\
\hline Worker or personnel in the service industry & 13 & 13 & & \\
\hline Scientific, educational and technical personnel & 12 & 27 & & \\
\hline Educational level & & & 6.685 & 0.004 \\
\hline Primary school & 21 & 1 & & \\
\hline Junior high school & 44 & 12 & & \\
\hline Senior high school (technical secondary school) & 26 & 23 & & \\
\hline Junior college & 10 & 14 & & \\
\hline Undergraduate and above & 4 & 15 & & \\
\hline Economic income (yuan) & & & 10.819 & $<0.001$ \\
\hline$\leq 2,000$ & 38 & 3 & & \\
\hline$>2,000-\leq 4,000$ & 48 & 24 & & \\
\hline$>4,000$ & 19 & 38 & & \\
\hline
\end{tabular}

ART, assisted reproductive technology.

\section{Patients and methods}

Case study. A total of 105 pregnant women (observation group) who underwent delivery and were ultimately diagnosed with PIH in The Affiliated Hospital of Xuzhou Medical University (Xuzhou, China) from February 2015 to February 2017 were retrospectively analyzed, and they were divided into two subgroups (mild and severe groups) according to the severity of the disease. In addition, 65 patients without symptoms in the same period were included as the healthy control group. Under the precondition of complying with the regulations on the medical record management and confidentiality principles, information on the clinical characteristics of pregnant women with PIH and laboratory tests were queried and collected through the The Affiliated Hospital of Xuzhou Medical University medical records. Conception methods of pregnant women are divided into two types [natural conception and assisted reproductive technology (ART) conception (including artificial insemination, ovulation therapy, external fertilization, embryo transplantation and intracytoplasmic sperm injection)]. All the included patients or their family members were informed of the study and signed the informed consent. This study was approved by the Ethics Committee of The Affiliated
Hospital of Xuzhou Medical University. The diagnostic criteria for relevant diseases referred to Razak et al (7) and Ishimitsu (8). Inclusion criteria were: patients with gestational week $>28$ weeks and systolic blood pressure $\geq 140 \mathrm{~mm} \mathrm{Hg}$ and/or diastolic blood pressure $\geq 90 \mathrm{~mm} \mathrm{Hg}$ for the same arm at least two times. Exclusion criteria were: i) patients with primary hypertension; ii) patients with PIH caused by diabetes mellitus, nephritis, heart disease and other internal medicine diseases; and iii) patients with smoking, drug addicts or other harmful habits.

Research methods. Basic data were compared between the observation and healthy control groups, including periodic antenatal care, age (the age in the last menstrual period), gravidity, parity, gestational week, mode of conception, occupation, educational level and economic income of the pregnant women.

Obstetrical data were compared between the observation and healthy control groups, including placental abruption and properties of amniotic fluid (clear, turbid or bloody).

Data of perinatal infants were compared between the observation and healthy control groups, including neonatal birth weight, neonatal department transfer, small for 
Table II. Comparisons of obstetric data of pregnant women between PIH and healthy control groups.

\begin{tabular}{|c|c|c|c|c|}
\hline Obstetric data & Observation group $(n=105)$ & Control group $(n=65)$ & $\chi^{2}$ & P-value \\
\hline Placental abruption & & & 7.593 & 0.003 \\
\hline Yes & $24(22.86)$ & $1(1.54)$ & & \\
\hline No & $81(77.14)$ & $64(98.46)$ & & \\
\hline Amniotic fluid & & & 16.073 & $<0.001$ \\
\hline Clear & $62(59.05)$ & $54(83.08)$ & & \\
\hline Turbid & $32(30.48)$ & $10(15.38)$ & & \\
\hline Bloody & $11(10.48)$ & $1(1.54)$ & & \\
\hline
\end{tabular}

$\mathrm{PIH}$, pregnancy-induced hypertension.

gestational age (SGA), neonatal asphyxia and survival rates of perinatal infants. The birth weight (g) and Apgar score (points) (Apgar score $\leq 7$ points at $1 \mathrm{~min}$ after birth indicating neonatal asphyxia, 4-7 points indicating mild asphyxia and 0-3 points indicating severe asphyxia) of neonates were recorded.

PAPP-A and VEGF levels of pregnant women were compared between the healthy control and observation groups at different severity degrees. Collection of serum samples of pregnant women occurred as follows: before admission, $6 \mathrm{ml}$ elbow venous blood was drawn and placed in a dry test tube. The supernatant was collected after centrifugation at $3,000 \mathrm{x}$ g for $15 \mathrm{~min}$ at $4^{\circ} \mathrm{C}$ and stored at $-20^{\circ} \mathrm{C}$. Enzyme-linked immunosorbent assay (ELISA) was carried out to detect PAPP-A and VEGF with Human Pappalysin-1/PAPP-A Quantikine ELISA Kit and Human VEGF Quantikine ELISA Kit (R\&D Systems). All operations were performed by dedicated staff strictly following the relevant instructions.

Changes in the serum PAPP-A and VEGF levels of pregnant women at different gestational weeks in both groups were compared. At 1 day in each week between 34-40 gestational weeks, $6 \mathrm{ml}$ elbow venous blood was drawn and placed in a dry test tube as described in the previous paragraph.

Statistical analysis. Statistical Product and Service Solutions (SPSS) 18.0 software (SPSS, Inc.) was adopted for data analysis. Enumeration data were expressed as mean \pm standard deviation (SD) and detected by t-test. One-way analysis of variance was carried out for for multiple comparisons with Dunnett's post hoc test. The constituent ratio and percentage were compared via the exact probability test $\left(\chi^{2}\right.$ test) of contingency tables. Binary unconditional logistic regression models were applied to analyze risk factors resulting in PIH. $\mathrm{P}<0.05$ was considered to indicate a statistically significant difference.

\section{Results}

Comparison of basic data between the two groups of pregnant women. There were statistical differences in regular antenatal care, gravidity, parity, occupation, educational level and economic income between the two groups of pregnant women $(\mathrm{P}<0.05)$. The regular antenatal care rate in the observation group was lower, and this group mainly consisted of primiparae and housewives with lower education levels and economic income.
However, there were no statistically significant differences in age, gestational week and conception method $(\mathrm{P}>0.05$; Table I).

Comparison of obstetric data between the two groups of pregnant women. The incidence rates of placental abruption as well as turbid and bloody amniotic fluid in the observation group were higher than those in the healthy control group. Statistical analysis revealed that the differences were statistically significant $(\mathrm{P}<0.05$; Table II).

Comparison of perinatal fetus outcomes between the two groups. The neonatal birth weight was lower in the observation group than that in the healthy control group, while the occurrence rates of neonatal department transfer, SGA, neonatal asphyxia and survival rates of perinatal infants were higher $(\mathrm{P}<0.05$; Table III).

Comparison of the levels of serum PAPP-A and VEGF between the two groups of pregnant women. The levels of serum PAPP-A (U/l) in the healthy control, observation (mild) and observation (severe) groups were $23.51 \pm 7.83,41.36 \pm 10.57$ and $72.81 \pm 14.29$, respectively, displaying an increasing trend and statistically significant differences in intergroup comparisons $(\mathrm{P}<0.05)$. The levels of VEGF (ng/l) in the healthy control, observation (mild) and observation (severe) groups were $73.78 \pm 15.32,47.45 \pm 11.02$ and $33.62 \pm 6.19$, respectively, showing a decreasing trend and statistically significant differences in intergroup comparisons $(\mathrm{P}<0.05$; Fig. 1$)$.

Changes in the levels of serum PAPP-A in the two groups of pregnant women at different gestational weeks. The levels of serum PAPP-A (U/1) in the observation group at 34-40 weeks were $48.57 \pm 14.63,43.28 \pm 13.46,42.16 \pm 15.51,45.89 \pm 12.83$, $49.72 \pm 12.68,42.15 \pm 16.34$ and $48.60 \pm 17.57$, respectively, while those in the healthy control group at 34-40 weeks were $13.25 \pm 2.14,16.57 \pm 2.83,20.08 \pm 3.26,21.24 \pm 1.82,22.46 \pm 3.58$, $21.80 \pm 2.76$ and $28.97 \pm 5.85$, respectively. It is evident that the levels of serum PAPP-A at 34-40 gestational weeks in the observation group were significantly higher than those in the healthy control group, clearly manifesting statistically significant differences $(\mathrm{P}<0.05$; Fig. 2A).

Changes in the levels of serum VEGF in the two groups of pregnant women at different gestational weeks. The levels of 
Table III. Comparison of perinatal outcomes between the two groups.

\begin{tabular}{|c|c|c|c|c|}
\hline Relevant indexes & Observation group $(n=105)$ & Control group $(n=65)$ & $\mathrm{t} / \chi^{2}$ & P-value \\
\hline Neonatal birth weight (g) & $2,487.15 \pm 604.53$ & $3,201.46 \pm 453.12$ & 9.548 & 0.002 \\
\hline Admission in the Neonatology Department [n (\%)] & & & 10.057 & $<0.001$ \\
\hline Yes & $43(40.95)$ & $5(7.69)$ & & \\
\hline No & $62(59.05)$ & $60(92.31)$ & & \\
\hline SGA & & & 11.158 & $<0.001$ \\
\hline Yes & $37(35.24)$ & $3(4.62)$ & & \\
\hline No & $68(64.76)$ & $62(95.38)$ & & \\
\hline Neonatal asphyxia [n (\%)] & & & 7.614 & 0.004 \\
\hline No & $85(80.95)$ & $63(96.83)$ & & \\
\hline Mild & $14(13.33)$ & $2(3.08)$ & & \\
\hline Severe & $6(5.71)$ & $0(0.00)$ & & \\
\hline Survival rate & & & 12.753 & $<0.001$ \\
\hline Stillbirth & $8(7.62)$ & $0(0.00)$ & & \\
\hline Live birth & 97 (92.38) & $65(100.00)$ & & \\
\hline
\end{tabular}

SGA, small for gestational age.
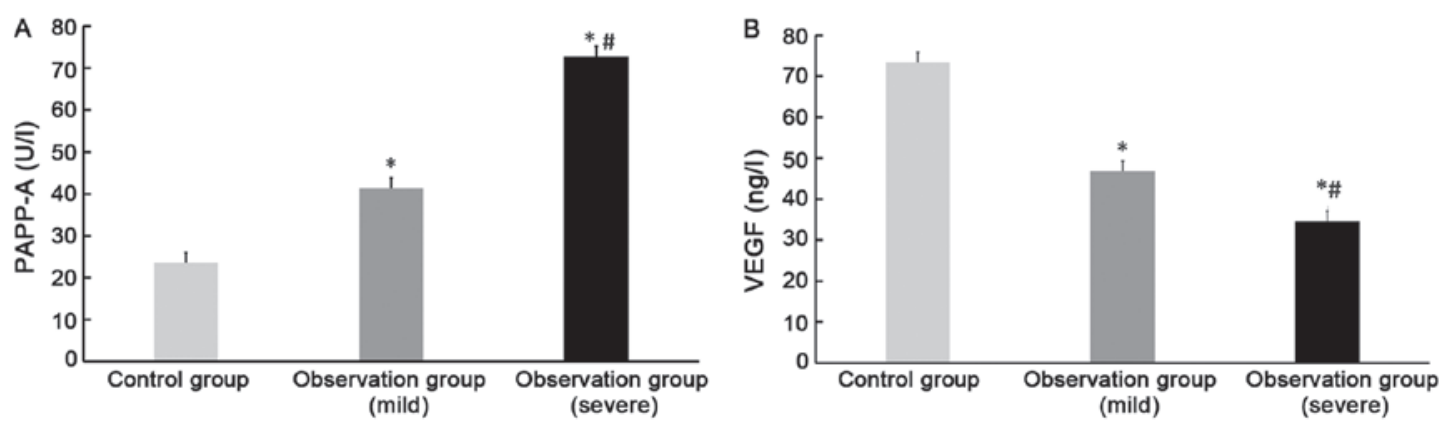

Figure 1. (A) Comparison of the levels of serum PAPP-A in pregnant women in the healthy control and observation groups with different severity degrees of the disease. (B) Comparison of the levels of serum VEGF in pregnant women in the healthy control and observation groups with different severity degrees of the disease. " $\mathrm{P}<0.05$ vs. the healthy control group. ${ }^{*} \mathrm{P}<0.05$ vs. the observation group (mild). PAPP-A, pregnancy-associated plasma protein A; VEGF, vascular endothelial growth factor.
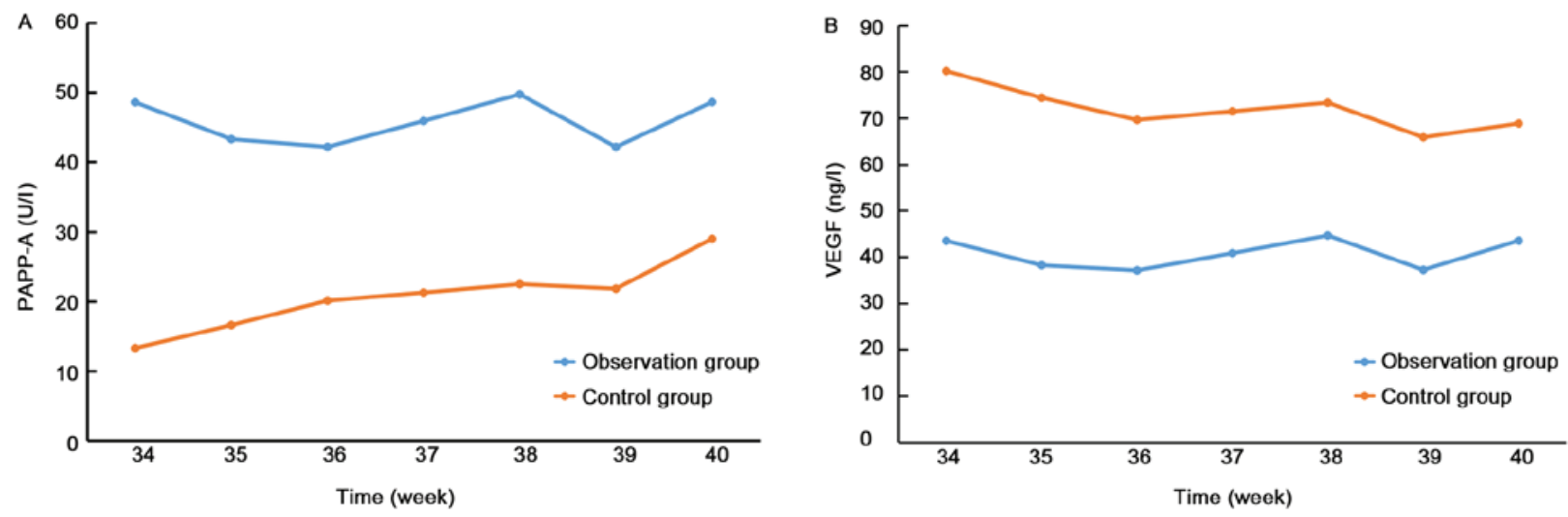

Figure 2. (A) Changes in the levels of serum PAPP-A in pregnant women at different gestational weeks in the two groups. (B) Changes in the levels of serum VEGF in pregnant women at different gestational weeks in both groups. The levels of serum VEGF at all gestational weeks in the observation group are evidently higher than those in the healthy control group $(\mathrm{P}<0.05)$. PAPP-A, pregnancy-associated plasma protein A; VEGF, vascular endothelial growth factor.

serum VEGF (ng/l) in the observation group at 34-40 weeks were $43.46 \pm 2.52,38.17 \pm 2.61,37.05 \pm 2.49,40.78 \pm 2.35$,
$44.61 \pm 2.57,37.16 \pm 2.43$ and $43.49 \pm 2.46$, respectively, while those in the healthy control group at 34-40 weeks were 
Table IV. Logistic stepwise regression analysis of high-risk factors for PIH.

\begin{tabular}{|c|c|c|c|c|c|c|}
\hline Variables & Regression coefficient & Standard error & Wald value & P-value & OR & $95 \% \mathrm{CI}$ \\
\hline Regular antenatal care & -3.148 & 1.596 & 4.973 & 0.029 & 1.201 & $1.091-2.814$ \\
\hline Gravidity & -4.163 & -2.614 & 5.302 & 0.020 & 2.173 & $1.034-4.925$ \\
\hline Economic income & -3.412 & -2.853 & 7.918 & 0.005 & 4.162 & $1.645-8.376$ \\
\hline Housewife & 0.967 & 2.046 & 5.142 & 0.017 & 2.514 & $1.203-4.538$ \\
\hline PAPP-A & 3.581 & 4.232 & 6.417 & 0.002 & 3.736 & $1.140-7.419$ \\
\hline VEGF & -5.145 & -2.976 & 8.285 & 0.001 & 5.258 & $2.034-10.183$ \\
\hline
\end{tabular}

PIH, pregnancy-induced hypertension; OR, odds ratio; CI, confidence interval; PAPP-A, pregnancy-associated plasma protein A; VEGF, vascular endothelial growth factor.

$80.14 \pm 8.26,74.35 \pm 7.51,69.57 \pm 6.48,71.42 \pm 8.19,73.28 \pm 7.41$ $65.81 \pm 7.63$ and $68.79 \pm 8.05$, respectively. This revealed that the levels of serum VEGF at 34-40 gestational weeks in the observation group were remarkably lower than those in the healthy control group, displaying a statistically significant difference $(\mathrm{P}<0.05$; Fig. 2B).

Logistic stepwise regression analysis of high-risk factors for $P I H$. Data of all groups in this study were analyzed using the unconditional Logistic regression model. Patients were divided according to whether they had PIH, and PIH was directly quantified by the values of 0 and 1 (the dependent variable $\mathrm{y}=0$ represented no $\mathrm{PIH}$, while $\mathrm{y}=1$ stood for the existence of $\mathrm{PIH})$. During the analysis, the binary variables were directly quantified through assignments with 0 and 1, whereas multiple ordered categorical variables were quantified through assignments with $1,2,3, \ldots$ to indicate their low-level to high-level attributes. In this study, 6 out of 11 factors (including dummy variables) that may influence the occurrence of PIH, including regular antenatal care, age, gravidity, parity, gestational week, conception method, occupation, educational level, economic income, amniotic fluid shape and laboratory test were selected into the model.

Binary logistic regression model analysis was carried out. Based on the assignment of the dependent variable, positive regression coefficient of the independent variable indicated the factor is a risk factor, and the higher quantitative value would more easily promote the occurrence of PIH. Otherwise, it was a protective factor suppressing the occurrence of PIH. The analysis results manifested that the major risk factors leading to the occurrence of PIH were PAPP-A value and the identity of housewives in a descending order. The protective factors impeding the occurrence of PIH were VEGF value, non-primary pregnancy, relatively high economic income and regular antenatal care in a descending order (Table IV).

\section{Discussion}

The PAPP-A gene, first detected in plasma of pregnant women, is located on chromosome 9q33.1. It encodes a high-molecular-weight zinc-binding metalloproteinase related to insulin-like growth factors (IGFs) $(9,10)$. PAPP-A in pregnant women is mainly produced by placental tissues. Its content increases steadily with the increase of gestational week until delivery, and it disappears at 6 weeks after delivery (11). Biological effects of PAPP-A mainly include: i) IGFs regulate cell division and differentiation, and maintain the normal growth of the fetus and placenta. ii) IGF-II plays a crucial role in the regulation of trophoblast cells and infiltration of uterine decidual tissues. iii) IGFs regulate the transportation of the three nutrients in syncytiotrophoblasts in the villi. According to this study, the levels of serum PAPP-A in pregnant women exhibit an increasing trend in the healthy control, mild PIH and severe PIH groups $(\mathrm{P}<0.05)$, and PAPP-A levels at 34-40 gestational weeks in the observation group were higher than those in the healthy control group $(\mathrm{P}<0.05)$. Besides, the multivariate analysis revealed that the high PAPP-A value [odds ratio $(\mathrm{OR})=3.736$ ) was a risk factor for $\mathrm{PIH}$, which is similar to the conclusion of study of Bersinger et al (4). It is known that placental dysplasia and insufficient infiltration of trophoblast cells are one of the important pathogenesis of preeclampsia. The low-level PAPP-A enables IGF to be in a binding state, thereby leading to inadequate binding of IGFs to receptors, and further resulting in placental maldevelopment and insufficient infiltration of trophoblast cells. However, placental dysplasia and placental ischemia and hypoxia influence the secretion of PAPP-A by the placenta, and they interact with each other. PAPP-A exerts a vital role in the pathogenesis and progression of PIH.

Located on chromosome 6p21.3, the human VEGF gene is a single gene consisting of 8 exons and 7 introns with a total length of $14 \mathrm{~kb}$, whose encoding product is highly conserved homologous dimer glycoprotein (34-45 kDa) (12). Biological functions of VEGF are chiefly constituted by proliferation and division of endothelial cells, angiogenesis, survival and migration of endothelial cells, vascular permeability, and migration of monocytes and macrophages (13-15). VEGF is a mitogen of endothelial cells with high efficiency and specificity, and it plays a role in paracrine and autocrine manner. In addition, VEGF stimulates the proliferation and division of endothelial cells, enhances vascular permeability, induces angiogenesis and promotes the release of nitrogen (NO) in trophoblast cells, and in turn, the expansion of uteroplacental vessels caused by $\mathrm{NO}$ is the prerequisite for the infiltration of trophoblast cells and endometrial revascularization (16). Changes in VEGF are synchronized with NO synthase activity, both are the highest in the first trimester of pregnancy (17). Studies have illustrated that VEGF exerts different regulatory effects on different 
tissues of the reproductive system. The placental vasculogenesis function is decreased in the third trimester of pregnancy, and VEGF mainly acts as vascular permeability factor, which is conducive to fetal growth (18). This study demonstrated that the levels of serum VEGF in the healthy control, mild PIH and severe PIH groups displayed a decreasing trend $(\mathrm{P}<0.05)$, and the levels of VEGF at 34-40 gestational weeks in PIH group were lower than those in the healthy control group $(\mathrm{P}<0.05)$. Additionally, the multivariate analysis manifested that the high VEGF value $(\mathrm{OR}=5.258)$ could be regarded as a protective factor for PIH, which is similar to the conclusion of the study of Tsatsaris et al (19). The basic pathological change in PIH is systemic vasospasm, and the elevated maternal blood pressure is directly induced by the increased peripheral resistance to vasospasm. During vasospasm, the systemic perfusion of all organs is decreased, and hypoxia and ischemia occur locally in the body, which suppresses the proliferation, differentiation and infiltration of trophoblast cells, causing shallow placental implantation and vascular remodeling obstacles, which are related to VEGF. Thus, VEGF exerts a vital effect on the pathogenesis and progression of PIH.

In this study, Logistic regression screening was carried out for 11 factors that may affect the occurrence of PIH, including regular antenatal care, age, gravidity, parity, gestational week, conception method, occupation, education level, economic income, amniotic fluid shape and laboratory tests, and there were 6 factors selected into the model. The high PAPP-A value $(\mathrm{OR}=3.736)$ and identity of housewife $(\mathrm{OR}=2.514)$ were risk factors for $\mathrm{PIH}$, while the high VEGF value $(\mathrm{OR}=5.258)$, non-primary pregnancy $(\mathrm{OR}=2.173)$ and relatively high economic income $(\mathrm{OR}=4.162)$, regular antenatal care $(\mathrm{OR}=1.201)$ were regarded as protective factors. Housewives have high tendency to suffer from PIH and are a high-risk group for PIH. This may be because their central position in the family economy requires more concerns on pregnancy, child's birth and rearing, and other economic problems, which invisibly increase pressure and psychological tension. This is consistent with the report of Dekker (20). According to this study, women with primary pregnancy were more prone to $\mathrm{PIH}$ compared with women with multiple pregnancies, so the former became a high-risk group for PIH, which is consistent with previous reports (21). The immunological theory of PIH has indicated that pregnancy refers to a successful natural allograft (22), and the establishment and stabilization of the immune balance between maternal and fetal are prerequisites for the maintenance of normal pregnancy. The body of women with primary pregnancy lacks 'blocking antibodies', so they cannot resist the burden of fetal antigen, thus resulting in PIH (23). The incidence rates of placental abruption as well as turbid and bloody amniotic fluid in the PIH group were higher than those in the healthy control group $(\mathrm{P}<0.05$ in all comparisons). Besides, PIH group had lower neonatal birth weight and survival rate of perinatal infant but higher incidence rates of neonatal department transfer, SGA and neonatal asphyxia ( $\mathrm{P}<0.05$ in all comparisons), which is consistent with previous reports $(24,25)$.

This study manifested that the high PAPP-A value and the identity of housewife were main risk factors for PIH, while the high VEGF value, non-primary pregnancy, higher economic income, and regular antenatal care were its protective factors.
Hence, obstetricians should carry out a health campaign and education and emphasize the importance of regular antenatal care while they improve their overall levels of professional diagnosis and treatment. PAPP-A and VEGF levels should be monitored during pregnancy, and housewives, low-income families, women with primary pregnancy and other high-risk groups should be intensively monitored. The enhancement of pregnancy management, early detection and early correction are the keys to improving the prognosis of pregnant women and children.

\section{Acknowledgements}

Not applicable.

\section{Funding}

This study was supported by Jiangsu Province, Xuzhou City to promote the post-subsidy filing project of the Special Fund for Science and Technology Innovation (no. KH17045). Maternal and Child Health Research Project in Jiangsu Province (no. FYX201811).

\section{Availability of data and materials}

The datasets used and/or analyzed during the current study are available from the corresponding author on reasonable request.

\section{Authors' contributions}

$\mathrm{ZZ}$ wrote the manuscript. ZZ and HX collected and analyzed the basic data of patients. XL and PL were responsible for obstetrical data analysis. WD and QH performed ELISA. All the authors read and approved the final manuscript.

\section{Ethics approval and consent to participate}

The study was approved by the Ethics Committee of The Affiliated Hospital of Xuzhou Medical University (Xuzhou, China) and informed consents were signed by the patients or the guardians.

\section{Patient consent for publication}

Not applicable.

\section{Competing interests}

The authors declare that they have no competing interests.

\section{References}

1. Perveen S: Frequency and impact of hypertensive disorders of pregnancy. J Ayub Med Coll Abbottabad 26: 518-521, 2014

2. Duley L: Maternal mortality associated with hypertensive disorders of pregnancy in Africa, Asia, Latin America and the Caribbean. Br J Obstet Gynaecol 99: 547-553, 1992.

3. Smith GC, Stenhouse EJ, Crossley JA, Aitken DA, Cameron AD and Connor JM: Early pregnancy levels of pregnancy-associated plasma protein A and the risk of intrauterine growth restriction, premature birth, preeclampsia, and stillbirth. J Clin Endocrinol Metab 87: 1762-1767, 2002. 
4. Bersinger NA, Smárason AK, Muttukrishna S, Groome NP and Redman CW: Women with preeclampsia have increased serum levels of pregnancy-associated plasma protein A (PAPP-A), inhibin A, activin A and soluble E-selectin. Hypertens Pregnancy 22: 45-55, 2003.

5. Svedas E, Islam KB, Nisell H and Kublickiene KR: Vascular endothelial growth factor induced functional and morphologic signs of endothelial dysfunction in isolated arteries from normal pregnant women. Am J Obstet Gynecol 188: 168-176, 2003.

6. Luizon MR, Sandrim VC, Palei AC, Lacchini R, Cavalli RC, Duarte G and Tanus-Santos JE: Epistasis among eNOS, MMP-9 and VEGF maternal genotypes in hypertensive disorders of pregnancy. Hypertens Res 35: 917-921, 2012.

7. Razak A, Florendo-Chin A, Banfield L, Abdul Wahab MG, McDonald S, Shah PS and Mukerji A: Pregnancy-induced hypertension and neonatal outcomes: a systematic review and meta-analysis. J Perinatol 38: 46-53, 2018.

8. Ishimitsu T: Starting the new review series: Pregnancy-induced hypertension. Hypertension Research 40: 3-4, 2017.

9. Silahtaroglu AN, Tümer Z, Kristensen T, Sottrup-Jensen L and Tommerup N: Assignment of the human gene for pregnancy-associated plasma protein A (PAPPA) to 9q33.1 by fluorescence in situ hybridization to mitotic and meiotic chromosomes. Cytogenet Cell Genet 62: 214-216, 1993.

10. Lin TM, Galbert SP, Kiefer D, Spellacy WN and Gall S: Characterization of four human pregnancy-associated plasma proteins. Am J Obstet Gynecol 118: 223-236, 1974.

11. Folkersen J, Grudzinskas JG, Hindersson P, Teisner B and Westergaard JG: Pregnancy-associated plasma protein A: circulating levels during normal pregnancy. Am J Obstet Gynecol 139: 910-914, 1981.

12. Jussila L and Alitalo K: Vascular growth factors and lymphangiogenesis. Physiol Rev 82: 673-700, 2002.

13. Rak J, Yu JL, Klement G and Kerbel RS: Oncogenes and angiogenesis: Signaling three-dimensional tumor growth. J Investig Dermatol Symp Proc 5: 24-33, 2000.

14. Duyndam MC, Hilhorst MC, Schlüper HM, Verheul HM, van Diest PJ, Kraal G, Pinedo HM and Boven E: Vascular endothelial growth factor-165 overexpression stimulates angiogenesis and induces cyst formation and macrophage infiltration in human ovarian cancer xenografts. Am J Pathol 160: 537-548, 2002.

15. Kerbel RS: Tumor angiogenesis. N Engl J Med 358: 2039-2049, 2008 .
16. Nanaev A, Frank HG, Chwalisz K and Kaufmann P: Physiological dilatation of uteroplacental arteries in the guinea pig is related to nos-activity of invasive trophoblast. Placenta 16: A51, 1995.

17. Rutherford RA, McCarthy A, Sullivan MH, Elder MG, Polak JM and Wharton J: Nitric oxide synthase in human placenta and umbilical cord from normal, intrauterine growth-retarded and pre-eclamptic pregnancies. Br J Pharmacol 116: 3099-3109, 1995.

18. Lam PM, Po LS, Cheung LP and Haines C: The effect of exogenous estradiol treatment on the mRNA expression of vascular endothelial growth factor and its receptors in cultured human oviduct mucosal cells. J Assist Reprod Genet 22: 251-255, 2005.

19. Tsatsaris V, Goffin F, Munaut C, Brichant JF, Pignon MR, Noel A, Schaaps JP, Cabrol D, Frankenne F and Foidart JM: Overexpression of the soluble vascular endothelial growth factor receptor in preeclamptic patients: pathophysiological consequences. J Clin Endocrinol Metab 88: 5555-5563, 2003.

20. Dekker GA: Risk factors for preeclampsia. Clin Obstet Gynecol 42: 422-435, 1999.

21. Abi-Said D, Annegers JF, Combs-Cantrell D, Frankowski RF and Willmore LJ: Case-control study of the risk factors for eclampsia. Am J Epidemiol 142: 437-441, 1995.

22. Schuiling GA and Faas MM: Etiology and pathogenesis of preeclampsia: current concepts. Am J Obstet Gynecol 181: 1036-1037, 1999.

23. Robillard PY, Dekker GA and Hulsey TC: Revisiting the epidemiological standard of preeclampsia: Primigravidity or primipaternity? Eur J Obstet Gynecol Reprod Biol 84: 37-41, 1999.

24. Hoff C, Peevy K, Giattina K, Spinnato JA and Peterson RD: Maternal-fetal HLA-DR relationships and pregnancy-induced hypertension. Obstet Gynecol 80: 1007-1012, 1992.

25. Sletnes KE, Wisløff F, Moe N and Dale PO: Antiphospholipid antibodies in pre-eclamptic women: relation to growth retardation and neonatal outcome. Acta Obstet Gynecol Scand 71: 112-117, 1992.

This work is licensed under a Creative Commons Attribution-NonCommercial-NoDerivatives 4.0 International (CC BY-NC-ND 4.0) License. 